\title{
Case report of disseminated nocardiosis during immunosppressive treatment
}

\author{
Maria Teresa Gallo', Grazia Prignano', Pietro Donati², Elena Greco', Alan Di Maio', Chiara Pascolini', \\ Laura Cilli', Alessandra De Santis', Matteo Vespaziani', Fabrizio Ensoli' \\ I S.C. Patologia Clinica e Microbiologia \\ 2 U.O.S.D. Diagnostiche Microscopiche e Ultrastrutturali \\ Istituto Dermatologico San Gallicano, IRCCS, ROMA
}

Key words: Nocardia , immunocompromised, sulphamethoxazole, trimethoprim

Caso clinico di Nocardiosi disseminata durante trattamento immunosoppressivo

\section{SUMMARY}

Infection caused by Nocardia spp. Is unusual and presents with a variety of clinical manifestations in both immunocompromised patients and in immunocompetent hosts. The diagnosis of Nocardia is often difficult, and further hampered by the absence of specific clinical symptoms. A man, 63 years old, caring for about a month from multiple erosions of the oral cavity and Inca intergluteale associated with blisters and erosions scattered in the face, scalp and trunk. The routine laboratory tests, including serological testing for HIV and the determination of circulating CD4 lymphocytes, were negative. The chest X-ray was also negative. Due to the diagnostic suspicion of Pemphigus vulgaris (PV), therapy with methylprednisolone and azathioprine was initiated. After 15 days the patient's condition worsened and it became necessary hospitalization in intensive care. A CT scan found: pleural empyema, abscess, and paravertebral muscle abscess. The cultural examination of pleural fluid and synovial fluid gave positive results for Nocardia asteroides. On this basis, sulphamethoxazole was administered in association with trimethoprim for 2 months, with a complete remission of the symptoms. At a median follow up of 2 years the patient's general condition was excellent, without residual signs of infection. The cultured samples from pleural and synovial fluids gave a clear positivity after 5 days of incubation at $37^{\circ} \mathrm{C}$ on blood agar and chocolate agar plates; the colonies showed chalky and thin filaments, slide as branched Gram-positives and appeared partially acid-alcohol resistant by modified Kinyoun staining $\left(1 \% \mathrm{H}_{2} \mathrm{SO} \mathrm{O}_{4}\right)$. The presumptive identification of Nocardia spp. was additionally confirmed by biochemical tests for the identification of species with the following results: catalase test positive, oxidase test negative, aesculin hydrolysis, reduction of nitrite to nitrate and urease test positive. Given the low incidence of Nocardia spp. infection, and the non-typical clinical manifestations, microbiological diagnosis of this infection is critical for the selection of an appropriate antibiotic therapy that should be prolonged for several months.

L’infezione causata da Nocardia spp. si presenta con una varietà di manifestazioni cliniche spesso di difficile interpretazione sia in pazienti immunodepressi sia in pazienti immunocompetenti (1). Può causare malattie polmonari, lesione cutanee o patologie sistemiche con il coinvolgimento del sistema nervoso centrale (5). È tuttavia frequente la colonizzazione asintomatica delle vie aeree. Anche se l' infezione da Nocardia spp. può interessare individui sani, il rischio maggiore è presente in pazienti con compromissione della immunità cellulo-mediata, come nel caso di pazienti oncologici, soggetti con immunodeficienza congenita o acquisita, o pazienti in trattamento immunosoppressivo (7).

L'individuazione di una "nocardiosi" è spesso clinicamente complicata dalla assenza di una sintomatologia specifica che ne indirizzi la diagnosi(1).

Le Nocardie sono microrganismi catalasi-positivi, all'osservazione microscopica si presentano immobili, crescono in filamenti ramificati Gram +, spesso alcool acido-resistenti. La crescita delle colture può richiedere da una a più settimane di incubazione. Un uomo di 63 anni, affetto da circa un mese da erosioni della cavità orale e dell'incavo intergluteale, associate a vescicole sparse ed erosioni al viso, cuoio capelluto e busto. I test di laboratorio di routine, incluso quello sierologico per la infezione da HIV e la determinazione dei linfociti T CD4 circolanti, hanno dato risultati nella norma. L'esame RX del torace era negativo. Nel sospetto diagnostico di Pemphigus vulgaris (PV), è stata iniziata una terapia con metilprednisolone ed azatioprina. Dopo 15 giorni le condizioni del paziente sono peggiorate e si è reso necessario il ricovero nel reparto di Terapia Intensiva. Una tomografia assiale computerizzata ha rilevato: empiema pleurico, ascesso paravertebrale e ascessi muscolari. L'esame colturale del liquido pleurico e del liquido sinoviale era positivo per Nocardia asteroides. È stato somministrato sulfometossazolo con trimethoprim per 2 mesi, con completa remissione della sintomatologia. Ad un follow up di 2 anni le condizioni generali del paziente erano eccellenti, in assenza di residui segni di infezione. È stata effettuata la coltura del liquido pleurico e del liquido sinoviale su Agar cioccolato e Agar sangue. L'aspetto morfologico delle colonie di 2-3 mm di diametro cresciute sul terreno Agar-cioccolato dopo una settimana di incubazione a $35^{\circ} \mathrm{C}$ e $5-10 \% \mathrm{CO}_{2}$, era rugoso con margini irregolari, inizialmente di apparenza biancastra, in seguito assumendo una tinta arancio (Figura1). L' esame microscopico dopo colorazione di Gram e di Kinyoun modificata $\left(1 \% \mathrm{H}_{2} \mathrm{SO}_{4}\right)$ ha rivelato la presenza di filamenti a forma bacillare e alcoolacido resistenti. La conduzione dei tests di identificazione di specie sono stati limitati dalla difficoltà a mantenere vitale il ceppo isolato, pertanto non si è potuto eseguire un algoritmo diagnostico completo. Tuttavia, in aggiunta all'osservazione morfologica e microscopica delle colonie sono state eseguite le seguenti reazioni biochimiche $(3,6)$ :

- Positività al test dalla catalasi eseguito con perossido di ossigeno al 3\%,

- Negatività al test dell'ossidasi (Mast-ID,Mast,UK)

- Positività alla idrolisi dell'esculina (Ferric Ammonium Citrate, BBL): l'esculina reagisce con sale di ferro contenuto nel terreno e produce una colorazione nera. Per questo test abbiamo utilizzato, come controllo positivo, il ceppo di Nocardia farcinica (ATCC 3318). L’incubazione è stata protratta per 14 giorni ad una temperatura di $35^{\circ} \mathrm{C}$.

- Positività al test dell'ureasi: l'agar (Urea Agar Slant, Nitrate Broth, BBL) è stato inoculato e incubato a temperatura ambiente per due settimane. La Nocardia, contenendo ureasi, ha causato la riduzione da nitrati a nitriti con conseguente viraggio del terreno al rosso. Il ceppo di Nocardia brasiliensis (ATCC 19296) è stato utilizzato come controllo positivo.

Questo caso evidenzia l'importanza di una approfondita indagine sulla situazione clinica del paziente per indirizzare

\section{Corresponding author: Maria Teresa Gallo}

00144 Roma - Via Elio Chianesi, 53

Telefono: 0652666966 - Fax: 0652666II8

E-mail: gallo@ifo.it 


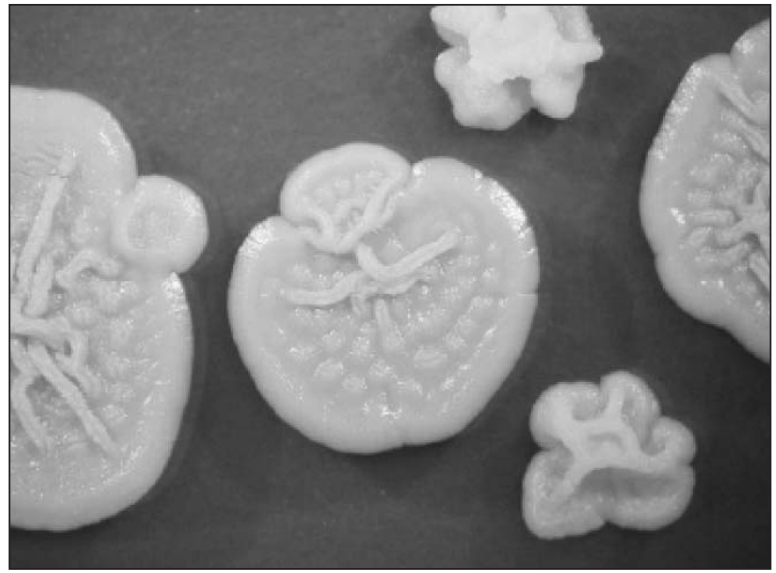

Figura I. Nocardia asteroides su Agar Cioccolato

una diagnosi microbiologica precoce e mirata. In questo caso, la presenza di empiema pleurico e ascessi multipli in corso di terapia immunosoppressiva, somministrata al paziente per la patologia bollosa autoimmune, ha indotto ad indirizzare la ricerca microbiologica anche verso il genere degli Actinomiceti. L'avvio del trattamento antibiotico appropriato (solfometazolo $400 \mathrm{mg}$ associato a trimethoprim $80 \mathrm{mg}$, tre volte al giorno, per due mesi) ha portato il pazien- te alla guarigione clinica dall'infezione. Il trattamento con metil- prednisolone, $0.3 \mathrm{mg} / \mathrm{pro}$ die, per il controllo della concomitante patologia bollosa autoimmune (Pemfigo Volgare) è stato peraltro mantenuto senza segni clinici di infezioni recidive nei due anni di follow-up. Non sono stati quindi necessari controlli microbiologici successivi alla prima identificazione.

\section{BIBLIOGRAFIA}

1. Agterof MJ, van der Bruggen T, Tersmette M, ter Borg EJ, van den Bosch MM, Biesma DH. Nocardiosis: a case series and a mini review of clinical and microbiological features. Neth J Med. 2007 Jun; 65(6): 199-202.

2. Bernadete M, Chedid F, Marcio F. Chedid, Nelson S. Porto, et al. Nocardial infections: report of 220cases Rev Inst Med trop Jul/Aug 2007.

3. Clinical Microbiology Procedures Handbook Henry D. Isenberg 2004.

4. Deanna LK, Hicks K, Pettit DJ. Identification of Medically Relevant Nocardia Species with an Abbreviated Battery of Testas. J Clin Microbiology. Apr.2002; 1346-51.

5. Dias M, Nagarathna S, Mahadevan A, Chandramouli BA, Chandramuki A. Nocardial brain abscess in an immunocompetent host. Indian $J$ Med Microbiol. 2008 Jul-Sep; 26(3): 274-7.

6. Luis M, de la Maza, Maria T, Pezzlo Janet, et al. Atlante a colori di Batteriologia medica 2006.

7. Mrozek N, Hamizi S, Gourdon F, Laurichesse H, Beytout J, Lesens O. Potential nosocomial disseminated infection due to Nocardia asteroides after a prosthesis insertion in an immunocompetent patient. Rev Med Interne. 2008 Dec; 29(12): 1034-7.

8. Por M, Barrio IA, Martinez MC, et al. Isolation of Nocardia Species Patients With Cys Fibrosos Arch Bronconeumol. 2008; 44: 109-12.

9. Schlaberg R, Huard RC, Della Latta P. Nocardia cyriacigeorgia, an Emerging Pathogen in the United States. Journal of Clinical Microbiology. Jan 2008: 265-73. 